\title{
Lymphangiogenesis and Anti-lymphangiogenesis in Cutaneous Melanoma
}

\author{
MARIUS RAICA, ADRIANA-ANDREEA JITARIU and ANCA MARIA CIMPEAN \\ Department of Microscopic Morphology/Histology, Angiogenesis Research Center \\ "Victor Babes" University of Medicine and Pharmacy, Timisoara, Romania
}

\begin{abstract}
Cutaneous malignant melanoma is an aggressive tumor characterized by early lymph node metastasis and bad prognosis. Although the spread of tumor cells in the regional lymph nodes is very important in the staging, prognosis and therapeutic strategy of malignant melanoma, the mechanism(s) of initial lymphatic vessels invasion is are) not completely understood. In the present review, we analyze the main factors involved in melanoma-associated lymphangiogenesis, based on existing available evidence. Currently, there are no anti-lymphangiogenic drugs approved for clinical trials. On the other hand, inhibition of lymph node metastasis has been demonstrated in experimental models by inhibiting tumor-associated lymphangiogenesis.
\end{abstract}

Despite the latest efforts concerning early detection, both the incidence and mortality caused by malignant melanoma of the skin, continue to grow. Malignant melanoma represents less that $10 \%$ of all cutaneous tumors, which is, however, responsible for most deaths caused by tumors of the skin. The natural evolution of malignant melanoma depends on its metastatic dissemination that is, in turn, influenced by multiple factors, particularly the thickness of the tumor, its localization and pathological subtype. Malignant melanomas of the skin are characterized, amongst other things, by early lymphatic dissemination in the regional lymph nodes and by lymph node metastases that significantly influence their staging, prognosis

This article is freely accessible online.

Correspondence to: Anca Maria Cimpean, MD, Ph.D., Professor of Histology, Department of Microscopic Morphology/Histology, Angiogenesis Research Center Timisoara, Pta Eftimie Murgu 2, 300041 Timisoara, Romania. Tel: +40 720060955, e-mail: ancacimpean1972@yahoo.com

Key Words: Lymphangiogenesis, lymph node metastasis, malignant melanoma, growth factors, prognosis, anti-lymphangiogenic therapy, review. and clinical approach. As a matter of fact, the detection of micrometastases in the sentinel lymph node is of prognostic value and represents the most important predictive criterion for survival in patients with no clinical evidence of lymph node metastases. Moreover, the global survival of patients lacking lymph node metastases is over $85 \%$ in a 10 -year follow-up and that of patients with positive lymph nodes is less than $45 \%$ (1). Once lymph node metastases become clinically obvious, 75$80 \%$ of patients already have other distant metastases. It is notable that $15 \%$ of the patients diagnosed with thin malignant melanoma (less than $1 \mathrm{~mm}$ ) develop metastases. At the time being, there are no highly specific predictive indicators for metastases development in patients diagnosed with melanoma. Although the clinical significance of lymph node metastases is well documented, the molecular mechanisms through which the primary tumor disseminates its malignant cells and the means through which these cells invade the lymphatic capillaries are rather less studied. Until recently, lymph node colonization with tumor cells was considered a passive process exclusively associated with the pre-existent lymphatic vessels. Currently, it is well-demonstrated that tumor cells and inflammatory cells contribute to tumor cell dissemination through the newly formed lymphatic vessels, a process known as lymphangiogenesis.

\section{Lymphangiogenesis}

Lymphangiogenesis represents the process through which new lymphatic vessels emerge from pre-existent vessels or post-capillary venules following the induction of the $P R O X$ 1 gene. In the majority of cases, this process occurs in pathologic inflammatory conditions and, especially, in tumor conditions. Lymphangiogenesis is induced and maintained by growth factors amongst which the most efficient are the vascular endothelial growth factor (VEGF)-C and VEGF-D that bind to their specific receptor, VEGFR-3, that is expressed by lymphatic endothelial cells (LECs). VEGF-C binding to VEGFR-3 induces LECs proliferation and 
formation of new lymphatic vessels in both the tumor and the peritumor areas. Considering the lymphatic dissemination pathway and the prognostic role along with the therapeutic impact of lymph node metastases, lymphangiogenesis is nowadays intensely studied in the majority of human tumors. Until now, the majority of published data support the fact that malignant tumor-associated lymphangiogenesis is a characteristic of poor prognosis and correlates with low global and disease-free survival (DFS).

\section{Lymphatic Vessels and Lymphangiogenesis in Malignant Melanoma}

Although lymphatic vessels have been documented since very long, the specific identification of the lymphatic endothelium is a relatively recent acquisition. The first markers with high specificity were characterized and purified less than two decades ago and, amongst them, podoplanin possesses the highest specificity and has the advantage of not staining the endothelial cells of the blood vessels. Currently, D2-40, the formalin-insensitive epitope of podoplanin, is the most frequently applied antibody for the immunohistochemical identification of LECs. The first indirect molecular identification of lymphatic vessels (LVs) in malignant melanoma dates back in 1990, when Fallowfield and Cook (2) applied a double immunohistochemical reaction for Ulex europeus 1 and collagen IV. The results were inconsistent, most probably generated by the absence of a specific marker and perhaps by the low number of cases included in the study $(n=21)$. The term lymphangiogenesis in malignant melanoma was introduced by de Waal et al. (3) (1997), although they did not manage to demonstrate the fact that this phenomenon actually existed. However, they did not investigate the proliferative potential of the lymphatic endothelium.

The first factual observation on lymphangiogenesis in malignant melanoma was made in an in vivo experimental study that used chick chorioallantoic membrane on which A375 melanic cells that expressed VEGF-C were implanted (4). It has been demonstrated that the A375 cell line induces the formation of numerous LVs at the level of the proliferation front, a large number of tumor cells invaded the lymphatic vessels and the proliferation rate of LECs identified with bromodeoxyuridine (BrdU) was calculated to be $11.6 \%$. In this model, lymphangiogenesis was significantly inhibited by VEGFR-3 blockage, with the proliferation index decreasing to $3.9 \%$. Also, the murine malignant melanoma cell line B16F10 implanted in the mouse induces the coordinated growth of blood and lymphatic vessels in the direction of the regional lymph nodes (5).

The presence of intratumor LVs and the enlargement of the peritumor ones were demonstrated in an animal experimental model by transplanting the VEGF-Coverexpressing human malignant melanoma in the nude mouse (6). Malignant melanoma is one of the first human tumors in which the existence of intratumor lymphatic vessels with a proliferative endothelium was demonstrated (7). As the refinement of technical methods increased, consistent results that partially explained the mechanisms of lymphatic vascular invasion and of lymph node metastases were obtained. From this point of view, VEGFR-3 expression was even considered a marker for the progression of malignant melanoma (8), a hypothesis partially exaggerated by the exclusion of other elements that characterized lymphangiogenesis.

\section{Mechanisms of Tumor Lymphangiogenesis}

The mechanisms of tumor lymphangiogenesis are complex and have generated several hypotheses that essentially reproduce certain stages of embryonic development. It has been demonstrated that LVs' proliferation in malignant tumors is dominantly dependent on the burgeoning of preexisting vessels and little or not at all on the insertion of bone marrow-derived progenitor endothelial cells (9). The mechanisms that induce malignant melanoma metastasis in the capillary vessels are uncertain, although lymph node metastases are a certainty. A series of hypotheses were issued on this concern: chemotaxis and LECs proliferation as a response to tumor cells or the fact that LECs secrete chemotactic agents for the tumor cells. Malignant melanoma cells express CCR7 receptors for CCL21 lymphatic chemokine and their interaction may contribute to LVs' invasion (10). CCR7 expression is correlated with the increase of VEGF-C and the decrease of the inflammationassociated interferon gamma and with a larger number of tumor cells that invade LVs. Consecutively, CCL21 expression increases in the lymphatic vessels in such a manner that this signaling pathway possesses a prolymphangiogenic effect in experimental models (11).

It has been demonstrated that LECs in culture may have a chemotactic laminin-421-mediated effect on tumor cells of malignant melanoma and that anti-laminin 421 antibodies annihilate this effects (12). These data suggest that laminin421 may mediate LVs' invasion but, up to this point, there are no available clinico-pathological observations to support this hypothesis. Another candidate substance in the LVs' invasion mechanism in melanomas is represented by alpha4beta1 integrin that induces lymphangiogenesis and stimulates the development of lymph node metastases in experimental models. The blockage of alpha-4-beta-1 integrin annihilates both effects in such a manner that it may be considered a potential therapeutic target (13).

Currently, several substances, such as erythropoietin that stimulates VEGF-C secretion in macrophages, have been characterized as possessing a pro-lymphangiogenic and prometastatic activity in experimental models; however, these 
observations require human specimens for validation (14). The mechanisms of lymphangiogenesis were also validated in an in vivo experimental model. Therefore, the implantation of B16 malignant cells line of pigmented melanoma that secrete VEGF-C generated the formation of vessels containing PROX-1 and VEGFR-3-expressing endothelial cells in the chick chorioallantoic membrane. The process was significantly evident under the combined influence of VEGF-A and hepatocyte growth factor (15). These results demonstrate that lymphangiogenesis induction and maintenance involve several factors that act successively or synchronously.

\section{Lymphovascular Invasion}

The identification of tumor cells in the lymphatic vessels of the primary tumor is of great prognostic value and represents a mandatory component of the histopathologic examination. On the other hand, lympho-vascular invasion in primary melanoma detected on conventionally stained sections is very low as compared to the incidence of metastases in the sentinel lymph node (16). Because of this, LVs' identification using specific markers for the lymphatic endothelium that improve diagnostic accuracy from $4.6 \%$ detected on hematoxylin-eosin stained slides to $43 \%$ on D2-40 stained slides, comes as a necessity. The immunohistochemical method based on D2-40, combined or not with S100 protein, identifies lympho-vascular invasion in a significantly larger number of patients than the routine staining methods $(10 / 27$ versus $1 / 27$ ) (17). Lymphatic invasion detected on D2-40 stained sections is associated with a deep Clark level, a high Breslow index and has a predictive role for metastases in the sentinel lymph node in $61 \%$ of cases $(18,19)$.

\section{Who is Invading Whom?}

Long has it been considered that tumor cells enter the lymphatic vessels through a passive process, generated by the discontinuities in the wall of the lymphatic capillaries. A lot of data that sustain LVs' invasion through the initiation of some molecular signaling pathways have currently been accumulated, a process that is more or less stimulated by growth factors and specific receptors. Microscopy observations on serial sections stained for the marker of the lymphatic endothelium alert the fact that the leader LECs (tip cells) present intratumor proliferation and, through branching, include smaller or larger tumor cell groups that in the early "tube-like" organization phase, remain included in the lumen. This process might be mediated via VEGF-C and VEGF-D, secreted by the malignant melanoma cells (unpublished data). Dynamic evaluations are not yet available on this incompletely demonstrated possible process. If it is true, then the leader cells may become an attractive target for the inhibition of early dissemination.

\section{Lymphatic Microvessel Density (LMVD)}

Until 10-12 years ago, lymphangiogenesis was associated with the density of the lymphatic microvessels from the tumor and peritumor area respectively. LMVD does not reflect other than the number of existing small lymphatic vessels at a certain point, in a certain tumor zone. On the other hand, numerous publications are available on this aspect of malignant melanoma, in the majority of cases high LMVD values being correlated with prognosis and, particularly, lymph node metastases. Also, in most studies, higher LMVD values were observed in the peritumor zone than the intratumor zone, along with significant morphologialc differences. The number of peritumor lymphatic vessels is significantly larger in cases of VEGF$\mathrm{C}$ and $-\mathrm{D}$ positive malignant melanomas than in the negative ones (10.05 vs. 6.4 and 10.07 vs. 6.3, respectively) (20). As a particularity, LMVD values may represent a discriminating aspect between metastatic malignant melanoma versus the non-metastatic one (12.8 and 5.9, respectively) (21). LMVD values are two-times higher in patients with metastatic melanoma affecting the sentinel lymph nodes compared to patients presenting negative lymph nodes $(22,23)$. Moreover, the number of peri- and intratumor LVs is greater in melanomas associated with metastasis in the sentinel lymph node than in the nonmetastatic melanoma (24). The data that have been published until now highlight the fact that increased LMVD values correlate with lymph node metastases and a shortterm survival. From this point of view, LMVD evaluation in melanomas constitutes a potential criterion in patient selection for complementary therapy and application of novel, targeted therapeutic strategies. A meta-analysis on LMVD in malignant melanoma has concluded that this evaluation possesses a prognostic value, along with lympho-vascular invasion. This statement is available in the case of peritumor LVs but not in that of intratumor ones (25). An overview of papers reporting data about LMVD is presented in Table I.

Different values between intra- and peritumor LMVD found by different authors mainly result from the means used in order to communicate the obtained results (square millimeters or per microscopic field), the marker applied for identifying endothelial lymphatic cells, as well as different methodologies used for LVs' counting.

The data regarding LMVD as a prognostic tool are still being debated and partially unclear. A recent study has proved that the Chalkey score applied for LVs' counting is an efficient predictor of metastases in the sentinel lymph node of distant metastases and global survival (33). Most studies that have included over 100 patients and were conducted using highly specific methods from a technical point of view, came up with these conclusions. 
Table I. Significance of lymphatic microvessel density (LMVD) in malignant melanoma.

\begin{tabular}{|c|c|c|c|c|}
\hline Authors & Year & Cases & Method & Significance \\
\hline Dadras et al. (26) & 2003 & 37 & LYVE-1/CD31 & Correlation with LNM and survival \\
\hline Straume et al. (27) & 2003 & 202 & LYVE-1 & Correlation with LNM \\
\hline Shields et al. (21) & 2004 & 21 & LYVE-1 & Correlation with LNM \\
\hline Valencak et al. (28) & 2004 & 120 & D2-40 & Correlation with LNM \\
\hline Dadras et al. (23) & 2005 & 45 & LYVE-1/CD31 & Correlation with LNM \\
\hline Sahni et al. (29) & 2005 & 36 & LYVE-1 & No correlation \\
\hline Massi et al. (24) & 2006 & 45 & D2-40 & Correlation with LNM and survival \\
\hline Liu et al. (20) & 2008 & 56 & LYVE-1 & Correlation with LNM \\
\hline Niakosari et al. (18) & 2008 & 96 & $\mathrm{D} 2-40$ & Correlation with LNM \\
\hline Emmett et al. (30) & 2010 & 102 & LYVE-1 & Correlation with LNM \\
\hline Storr et al. (31) & 2012 & 202 & $\mathrm{D} 2-40$ & No correlation \\
\hline Shayan et al. (32) & 2012 & 22 & D2-40 & Correlation with LNM \\
\hline
\end{tabular}

LNM, Lymph node metastases.

Shields index emerged from the observations on the prognostic values of LMVD, an index that is calculated on the basis of LMVD values, Breslow thickness and presence or absence of lympho-vascular invasion (LVI), with the latest being detected on sections stained by means of a specific marker for the lymphatic endothelium (21). The Shields index (SI) is calculated according to the following formula:

$\mathrm{SI}=\left(\mathrm{LMVD}^{2} \times\right.$ invasion $) \times$ Breslow thickness.

The mathematical calculation only considers the peritumor LMVD, detectable in over $90 \%$ of melanoma cases, where LVI is noted as 2 in case it is present and 1 in case it is absent. The Shields index is intensely correlated with the development of metastases in a 5-years interval $(\mathrm{SI}=2.43)$ in patients with metastases and 1.77 in those without metastases) (30).

\section{Growth Factors in Malignant Melanoma}

VEGF-C was evidenced in both tumor cells of primary malignant melanomas (34) and human malignant melanomaderived cell lines through mRNA and protein detection (35). VEGF-C was immunohistochemically detected in the cytoplasm of malignant cells in $70.97 \%$ of metastatic melanoma cases and $36 \%$ of non-metastatic cases (20). Current evidence demonstrate the correlation between VEGF-C overexpression in the primary tumor and existence of lymph node metastases (36). VEGF-C is not only secreted by the tumor cells but also tumor-associated macrophages. Its expression in the primary tumor, and particularly in thin melanomas, is correlated with the presence of metastases in sentinel lymph nodes; thus, the immunohistochemical identification of VEGF-C may be considered as a marker of tumor progression (37). The increased serum levels of VEGF-C, with values of over $1,600 \mathrm{pg} / \mathrm{ml}$, are significantly correlated with the presence of lymph node metastases. The exception is represented by patients under interferon alpha treatment in which case values are significantly lower (38). On the other hand, VEGF-C detection in primary melanoma of the skin is predictive for global and short-term DFS; however, it does not represent an independent prognostic survival factor (34). The mechanism through which VEGF$\mathrm{C}$ induces the formation of novel lymphatic vessels is still incompletely elucidated and stromal tumor cells, such as macrophages, may contribute to this process. In melanomas, tumor-associated macrophages secrete interleukin-1 beta and TNF-alpha, both of them inducing VEGF-C expression (36). It is quite possible that other stromal cells, such as mast cells, also possess an inductive effect on lymphangiogenesis. The relationship between mast cells and lymphangiogenesis has not largely been studied; however, arguments are available regarding this matter in inflammatory and tumor affections $(39,40)$, most probably due to the capacity of these cells to synthesize VEGF.

VEGF-D was identified in tumor cells of malignant melanomas and intratumor vessels but not in vessels located at a distance from the tumor (41). VEGF-D is structurally similar to VEGF-C, with both being correlated with the presence of lymph node metastases, thus having a negative effect on patients' survival. At the protein level, VEGF-D is expressed by the tumor cells of malignant melanomas in $74.19 \%$ of the studied cases of metastatic melanoma and in $36 \%$ of cases in non-metastatic melanomas (20).

VEGF-C and VEGF-D expression is significantly more intense in primary melanomas associated with lymph node metastases compared to non-metastatic melanomas. This aspect is obvious in case of tumor cells, but not in case of 
stromal cells, such as macrophages and fibroblasts (42). Among these two vascular growth factors, only VEGF-C is correlated with the intratumor and peritumor lymphatic vessel desnity in melanomas. VEGF-C and -D detection at the protein level may be considered a useful factor for prognosis refinement in malignant melanoma.

Lymphangiogenesis induction does not only imply the participation of the two above mentioned growth factors. The inductive role of VEGF-A was documented on lymphangiogenesis in the lymph nodes even before metastases occurence (7). This aspect is not surprising as it is known that the role of VEGF-A in the induction and progression of tumor lymphangiogenesis has been already documented in other tumor types (43).

However, the lymphangiogenic phenotype of malignant melanomas is more complex, considering the fact that the defining elements for this entity are VEGF-C expression, expression of the platelet-derived growth factor (PDGF)-C and that of the MET proto-oncogene (44). The identification of the three markers may represent a screening protocol for the lymphangiogenic profile of malignant melanoma. Despite this aspect, the lymphangiogenic potential is heterogeneously distributed in the tumor cells of the same case of malignant melanoma and is associated with the $M E T$ proto-oncogene signaling. Recently, hepatocyte growth factor, fibroblast growth factor 2, insulin-like growth factor and epidermal growth factor (EGF) have been documented as possessing a pro-lymphangiogenic effect (45).

Of particular interest is the effect of EGF on lymphangiogenesis, including malignant melanoma cases. Bracher et al. (46) demonstrated that EGF has a pro-metastatic effect, with EGF serum levels being significantly higher in patients with metastases than in those without metastases. EGF belongs to a family of growth factor capable of activating the specific receptor EGFR. Even if the inductive effects of EGF on pro-lymphangiogenic factors have been demonstrated, its effects on tumor lymphangiogenesis remain to be elucidated. In vitro observations have shown that EGF knockdown in malignant melanoma cells decreases the migration capacity of these cells and determines a significant reduction of metastases in spontaneously metastasized xenotransplant in a mouse model. EGF induces the occurrence of LVs' sprouting in vitro, a phenomenon that is inhibited by $E G F$ knockdown. Also, EGF is positively correlated with VEGF-C and PROX-1 expressions in the human primary melanoma, although in vivo EGF knockdown significantly decreases VEGF-C expression. Thus, it can be pointed out that EGF seems to play an important role in malignant melanomaassociated lymphangiogenesis $(46,47)$.

VEGFR-3 is a receptor with high specificity for VEGF-C and $-\mathrm{D}$ and associated with their expression in tumor cells. VEGFR-3 was immunohistochemically demonstrated in tumor cells (in over $80 \%$ of metastasized cases and in only
$36 \%$ of non-metastatic cases), with its expression in melanoma being significantly more intense than in melanocytic naevi $(20,48,49)$. VEGF-C and VEGFR-3 coexpression leads to the hypothesis of the autocrine regulation of a signaling pathway that may induce lymphangiogenesis. Low serum levels of VEGFR-3 are positively correlated with DFS. Current available data do not characterize VEGFR-3 as an independent prognostic factor. However, VEGFR-3 has been proposed as a therapeutic target; however, up until now, there are no relevant available data concerning this aspect.

Neuropilin-2, the co-receptor for VEGFR-3, plays the role of VEGF-C and -D receptor, therefore being implicated in the development and progression of angiogenesis. Based on this hypothesis, neuropilin-2 expression in malignant melanoma was studied and demonstrated in $86 \%$ of primary melanoma cases and in $90 \%$ of metastatic cases (50). Current data support the interaction between neuropilin and melanoma tumor cells and, taking into account the large number of positive cases, it may be considered as a biomarker of great interest in diagnosis. Its diagnostic value is strongly supported by the fact that it is intensely expressed in primary melanomas and in metastatic ones EGF knockdown but not in the melanocytic nevus. Also, the genetic expression of neuropilin-2 detected by means of polymerase chain reaction (PCR) increases from naevi to melanoma and is correlated with the progression of the neoplastic lesion (51). These data point to the fact that the expression of neuropilin selects a subset of patients presenting a high risk of progression and an unfavorable prognosis.

\section{Lymphangiogenesis in the Lymph Node Precedes Metastasis}

Malignant tumors in general, and malignant melanoma in particular, may induce a premetastatic niche characterized by induction of vessel growth in the sentinel lymph node even before the occurrence of metastasis. Under these circumstances, lymphangiogenesis in the lymph node increases metastasis dissemination and points out that activated LVs represent a novel therapeutic target for both detection and therapeutic approach of metastases (52). On the other hand, the characterization of activated LVs still remains unclear from a molecular point of view and further studies are required concerning this matter. At the time being, the only available methods are double immunostainings for D2-40/Ki67, highlighting LECs' proliferation, and D2-40/PROX-1 that pinpoint the lymphatic differentiation of the newly formed endothelial cells.

In a recent publication, Pastushenko et al. (53) showed that lymphangiogenesis and LECs' proliferation are more active in the primary tumor than in the lymph nodes. On the other hand, angiogenesis is activated in the lymph nodes, an 
Table II. Inhibitory substances of lymphangiogenesis in malignant melanoma experimental models.

\begin{tabular}{lccc}
\hline Substance & Effects & Authors & Year \\
\hline Anti-VEGFR-3 & Reduces the number of LVs, does not affect tumor cells & Hoshida et al. $(57)$ & Kinet et al. $(59)$ \\
Prolactin & Reduces the number of LVs from the tumor and lymph nodes & Duong et al. $(60)$ & 2006 \\
Anti-SOX18 & Inhibits tumor growth and lymphangiogenesis & Niederleithner et al. $(62)$ & 2012 \\
Wnt1 & Anti-lyphangiogenic agent through VEGF-C suppression & Roh et al. $(63)$ & 2012 \\
Interferon alpha2b & Antitumor, antimetastatic, anti-lymphangiogenic effects & Heinz et al. $(64)$ & 2013 \\
Activin A & Anti-lymphangiogenic agent, pleiotropic effect on motility & & 2015 \\
\hline
\end{tabular}

VEGFR, Vascular endothelial growth factor receptor; LVs, lymphatic vessels.

aspect that is demonstrated by the high proliferation rate of endothelial cells identified using CD34/Ki67, and correlated with patient's global survival.

Tumor cells present the capacity to choose the location of their future metastasis, thus having distant effects from the primary tumor before they disseminate $(54,55)$. Lymphangiogenesis in the sentinel lymph node is associated with a high frequency to develop metastases in the distant lymph nodes and, also, with a high incidence of metastases in the bloodstream. From this perspective, it may be stated that lymphatic and hematogenous metastasis do not represent independent processes. The current hypothesis regarding this phenomenon proposes VEGF-A and VEGF-C secretion by the tumor cells that are afterwards transported in the sentinel lymph nodes where they induce lymphangiogenesis and determine the formation of the pre-metastatic niche. When tumor cells populate the lymph node, VEGF-A and VEGF$\mathrm{C}$ concentrations are again increased and transported towards the distant lymph nodes (45). Lymph node changes were initially observed at the level of the sinuses that, as it is wellknown, express VEGFR-3. Besides the sinus hyperplasia, the lymph nodes that drain VEGF-C are characterized by the presence of a global profile of the lymphatic vascular network associated with the depopulation of germinal centers but without presenting any changes in the number of lymphatic follicles (56). The potential impact of this process on the therapeutic strategy is not yet exploited; however, a potential therapy based on VEGFR-3 inhibitors may be considered.

\section{Anti-lymphangiogenesis: Relations and Perspectives}

In the last decade, the Food and Drug Administration approved a series of anti-angiogenic substances for current application in human pathology. Amongst them, the most popular is bevacizumab, initially accepted as a therapeutic agent in colorectal cancer associated with hepatic metastases. Until now, numerous anti-angiogenic substances have been characterized but very few anti-lymphangiogenic substances have been taken into consideration. This problem may be explained by only few studies on lymphangiogenesis, incomplete characterization of therapeutic targets and, possibly, lack of knowledge regarding the microenvironment in which LVs develop and of the other cells that are implicated in this process.

The lack of an anti-lymphangiogenic medication remains a disappointing issue. One of the first trials in this field was conducted by Hoshida et al. (57) who studied the antiVEGFR-3 effect in malignant melanoma. The above mentioned authors observed the inhibition of novel LVs' formation but did not find any significant effects on tumor cells. On the other hand, the reduction of lympho-vascular invasion noticed rendered the VEGF-C/VEGFR-3 axis a therapeutic target, especially in the initial phases of lymphatic invasion. The usage of a recombined parvovirus as a sVEGFR3-Fc transporter showed that the stable systemic expression of sVEGFR3-Fc, followed by genetic transfer, markedly inhibits lymphangiogenesis and the development of metastases in the lymph nodes in malignant melanomas with a high metastatic potential (58). In the mentioned study, the sVEGFR3-Fc serum levels required for the efficient blocking of lymph node metastases were intensely dependant on the VEGF-C quantity generated by the primary tumor.

The angiostatic $\mathrm{N}$ terminal $16 \mathrm{kDa}$ fragment of human prolactin has proved to possess an antitumor effect. In vivo studies have demonstrated that this fragment of prolactin induces apoptosis and inhibits the proliferation, migration and formation of tubes by the microvascular dermal LECs. Also, in the experimental B16F10-induced melanoma model, the number of lymphatic vessels is significantly decreased in the primary tumor and lymph nodes following treatment based on the angiostatic fragment of prolactin (59). The mentioned study demonstrated the lymphangiogenic effects of prolactin for the first time and this observation may bring interesting perspectives on the general therapeutic strategy.

Relatively recent data have demonstrated that SOX18 is of critical importance for tumor proliferation, development 
of angiogenesis and lymphangiogenesis, through PROX-1 trans-activation in the lymphatic endothelial cells. A malignant melanoma experimental model proved that SOX18 inhibition has an inhibiting effect on both tumor growth and lymphangiogenesis and metastasis. From this point of view, SOX18 represents an attractive therapeutic target; however, it but requires validation and screening for potential inhibitory substances (60). Few of these substances, possessing an anti-lymphangiogenic effect, are presented in Table II. Some of them, such as activin A, have an anti-lymphangiogenic effect in experimental models but do not constitute a therapeutic target due to their side-effects.

Recently, a particular aspect was reported by Liersch et al. (61) who demonstrated the pro-lymphangiogenic in vitro effect of osteopontin. From its discovery in the bone tissue, osteopontin was intensely studied in different tumor types, with current results indicating its implication in determining its metastatic potential. Osteopontin expression stimulates lymphatic endothelial cell survival and migration, tumor growth and metastasis, therefore having the potential of becoming a promising candidate for targeted therapy in malignant melanoma.

An attractive therapeutic target is represented by the antipodoplanin antibodies. NZ-1, the first antibody of this series, was tested in an experimental model and proved to be a strong inhibitor of lymphangiogenesis and metastasis development (65). On the other hand, although specific for lymphatic endothelial cells, podoplanin is also expressed by certain normal cells, such as myofibroblasts, basal cells of the covering epithelia or by the reserve cells of sebaceous glands. The desideratum may be represented by an antibody with high specificity only for the activated lymphatic endothelial cells from the tumor microenvironment.

Considering the currently existing data in literature, no potentially applicable anti-lymphangiogenic medication can be recommended for malignant melanoma patients. Numerous studies are still necessary in order to accurately characterize the targets and mechanisms of initiation, as well as maintenance of melanoma-associated tumor lymphangiogenesis.

\section{Future Directions and Perspectives}

Since no anti-lymphangiogenic drugs are available for the clinical practice, further investigations of melanomaassociated lymphangiogenesis are necessary. On one hand, the characteristics of the lymphangiogenic potential of both malignant and stromal cells should be better standardized. Alternatively, the molecular profile of newly-formed lymphatic endothelial cells must be refined in order to characterize the target(s). Hopefully, new experimental models of melanoma-associated lymphangiogenesis will bring about new data on agents that could efficiently inhibit the proliferation of lymphatic vessels, lymphovascular invasion and lymph node metastasis.

\section{References}

1 Stadelmann WK, McMasters K and Polk HC Jr.: Diagnosis and treatment of cutaneous melanoma of the head and neck. J Ky Med Assoc 96: 46-55, 1998.

2 Fallowfield ME and Cook MG: Lymphatics in primary cutaneous melanoma. Am J Surg Pathol 14: 370-374, 1990.

3 de Waal RM, van Altena MC, Erhard H, Weidle UH, Nooijen PT and Ruiter DJ: Lack of lymphangiogenesis in human primary cutaneous melanoma. Consequences for the mechanism of lymphatic dissemination. Am J Pathol 150: 1951-1957, 1997.

4 Papoutsi M, Siemeister G, Weindel K, Tomarev SI, Kurz H, Schächtele C, Martiny-Baron G, Christ B, Marmé D and Wilting J: Active interaction of human A375 melanoma cells with the lymphatics in vivo. Histochem Cell Biol 114: 373-385, 2000.

5 Ruddell A, Croft A, Kelly-Spratt K, Furuya M and Kemp CJ: Tumors induce coordinate growth of artery, vein, and lymphatic vessel triads. BMC Cancer 14: 354, 2014.

6 Skobe M, Hamberg LM, Hawighorst T, Schirner M, Wolf GL, Alitalo $\mathrm{K}$ and Detmar $\mathrm{M}$ : Concurrent induction of lymphangiogenesis, angiogenesis, and macrophage recruitment by vascular endothelial growth factor-C in melanoma. Am J Pathol 159: 893-903, 2001.

7 Tobler NE and Detmar M: Tumor and lymph node lymphangiogenesis - impact on cancer metastasis. J Leukoc Biol 80: 691-696, 2006.

8 Clarijs R, Schalkwijk L, Hofmann UB, Ruiter DJ and de Waal RM: Induction of vascular endothelial growth factor receptor-3 expression on tumor microvasculature as a new progression marker in human cutaneous melanoma. Cancer Res 62: 70597065, 2002.

9 He Y, Rajantie I, Ilmonen M, Makinen T, Karkkainen MJ, Haiko $\mathrm{P}$, Salven $\mathrm{P}$ and Alitalo K: Preexisting lymphatic endothelium but not endothelial progenitor cells are essential for tumor lymphangiogenesis and lymphatic metastasis. Cancer Res 64: 3737-3740, 2004.

10 Shields JD, Emmett MS, Dunn DB, Joory KD, Sage LM, Rigby H, Mortimer PS, Orlando A, Levick JR and Bates DO: Chemokine-mediated migration of melanoma cells towards lymphatics - a mechanism contributing to metastasis. Oncogene 26: 2997-3005, 2007.

11 Takekoshi T, Fang L, Paragh G and Hwang ST: CCR7expressing B16 melanoma cells downregulate interferon- $\gamma$ mediated inflammation and increase lymphangiogenesis in the tumor microenvironment. Oncogenesis 1:e9. doi: 10.1038/oncsis.2012.9, 2012.

12 Saito N, Hamada J, Furukawa H, Tsutsumida A, Oyama A, Funayama E, Saito A, Tsuji T, Tada M, Moriuchi T and Yamamoto Y: Laminin-421 produced by lymphatic endothelial cells induces chemotaxis for human melanoma cells. Pigment Cell Melanoma Res 22: 601-610, 2009.

13 Garmy-Susini B, Avraamides CJ, Schmid MC, Foubert P, Ellies LG, Barnes L, Feral C, Papayannopoulou T, Lowy A, Blair SL, Cheresh D, Ginsberg $M$ and Varner JA: Integrin alpha4beta1 signaling is required for lymphangiogenesis and tumor metastasis. Cancer Res 70: 3042-3051, 2010. 
14 Lee AS, Kim DH, Lee JE, Jung YJ, Kang KP, Lee S, Park SK, Kwak JY, Lee SY, Lim ST, Sung MJ, Yoon SR and Kim W: Erythropoietin induces lymph node lymphangiogenesis and lymph node tumor metastasis. Cancer Res 71: 4506-4517, 2011.

15 Cîmpean AM, Seclaman E, Ceausu R, Gaje P, Feflea S, Anghel A, Raica $M$ and Ribatti D: VEGF-A/HGF induce Prox-1 expression in the chick embryo chorioallantoic membrane lymphatic vasculature. Clin Exp Med 10: 169-172, 2010.

16 Xu X, Gimotty PA, Guerry D, Karakousis G and Elder DE: Lymphatic invasion as a prognostic biomarker in primary cutaneous melanoma. Methods Mol Biol 1102: 275-286, 2014.

17 Petitt M, Allison A, Shimoni T, Uchida T, Raimer S and Kelly B: Lymphatic invasion detected by D2-40/S-100 dual immunohistochemistry does not predict sentinel lymph node status in melanoma. J Am Acad Dermatol 61: 819-828, 2009.

18 Niakosari F, Kahn HJ, McCready D, Ghazarian D, Rotstein LE, Marks A, Kiss A and From L: Lymphatic invasion identified by monoclonal antibody D2-40, younger age, and ulceration: predictors of sentinel lymph node involvement in primary cutaneous melanoma. Arch Dermatol 144: 462-467, 2008.

19 Schmidt CR, Panageas KS, Coit DG, Patel A and Brady MS: An increased number of sentinel lymph nodes is associated with advanced Breslow depth and lymphovascular invasion in patients with primary melanoma. Ann Surg Oncol 16: 948-952, 2009.

20 Liu B, Ma J, Wang X, Su F, Li X, Yang S, Ma W and Zhang Y: Lymphangiogenesis and its relationship with lymphatic metastasis and prognosis in malignant melanoma. Anat Rec (Hoboken) 291: 1227-1235, 2008.

21 Shields JD, Borsetti M, Rigby H, Harper SJ, Mortimer PS, Levick JR, Orlando A and Bates DO: Lymphatic density and metastatic spread in human malignant melanoma. Br J Cancer 90: 693-700, 2004.

22 Giorgadze TA, Zhang PJ, Pasha T, Coogan PS, Acs G, Elder DE and $\mathrm{Xu} \mathrm{X}$ : Lymphatic vessel density is significantly increased in melanoma. J Cutan Pathol 31: 672-677, 2004.

23 Dadras SS, Lange-Asschenfeldt B, Velasco P, Nguyen L, Vora A, Muzikansky A, Jahnke K, Hauschild A, Hirakawa S, Mihm MC and Detmar M: Tumor lymphangiogenesis predicts melanoma metastasis to sentinel lymph nodes. Mod Pathol 18 : 1232-1242, 2005.

24 Massi D, Puig S, Franchi A, Malvehy J, Vidal-Sicart S, González-Cao M, Baroni G, Ketabchi S, Palou J and Santucci M: Tumour lymphangiogenesis is a possible predictor of sentinel lymph node status in cutaneous melanoma: a case-control study. J Clin Pathol 59: 166-173, 2006.

25 Pastushenko I, Vermeulen PB, Carapeto FJ, Van den Eynden G, Rutten A, Ara M, Dirix LY and Van Laere S: Blood microvessel density, lymphatic microvessel density and lymphatic invasion in predicting melanoma metastases: systematic review and metaanalysis. Br J Dermatol 170: 66-77, 2014.

26 Dadras SS, Paul T, Bertoncini J, Brown LF, Muzikansky A, Jackson DG, Ellwanger U, Garbe C, Mihm MC and Detmar M: Tumor lymphangiogenesis: a novel prognostic indicator for cutaneous melanoma metastasis and survival. Am J Pathol 162: 1951-60, 2003.

27 Straume O, Jackson DG and Akslen LA: Independent prognostic impact of lymphatic vessel density and presence of low-grade lymphangiogenesis in cutaneous melanoma. Clin Cancer Res 9: 250-256, 2003.
28 Valencak J, Heere-Ress E, Kopp T, Schoppmann SF, Kittler H and Pehamberger H: Selective immunohistochemical staining shows significant prognostic influence of lymphatic and blood vessels in patients with malignant melanoma. Eur J Cancer 40: 358-364, 2004.

29 Sahni D, Robson A, Orchard G, Szydlo R, Evans AV and Russell-Jones R: The use of LYVE-1 antibody for detecting lymphatic involvement in patients with malignant melanoma of known sentinel node status. J Clin Pathol 58: 715-721, 2005.

30 Emmett MS, Symonds KE, Rigby H, Cook MG, Price R, Metcalfe C, Orlando A and Bates DO: Prediction of melanoma metastasis by the Shields index based on lymphatic vessel density. BMC Cancer 10: 208, 2010.

31 Storr SJ, Safuan S, Mitra A, Elliott F, Walker C, Vasko MJ, Ho B, Cook M, Mohammed RA, Patel PM, Ellis IO, NewtonBishop JA and Martin SG: Objective assessment of blood and lymphatic vessel invasion and association with macrophage infiltration in cutaneous melanoma. Mod Pathol 25: 493-504, 2012.

32 Shayan R, Karnezis T, Murali R, Wilmatt JS, Ashton MW, Taylor GI, Thompson JF, Hersey P, Achen MG, Scolyer RA and Stacker SA: Lymphatic vessel density in primary melanomas predicts sentinel lymph node status and risk of metastasis. Histopathology 61: 702-710, 2012.

33 Pastushenko I, Vermeulen PB, Vicente-Arregui S, Van den Eynden GG, Alvarez-Alegret R, Querol I, Rutten A, Carapeto FJ, Dirix LY and Van Laere S: Peritumoral D2-40 Chalkley score independently predicts metastases and survival in patients with cutaneous malignant melanoma. J Cutan Pathol 42: 699$711,2015$.

34 Boone B, Blokx W, De Bacquer D, Lambert J, Ruiter D and Brochez L: The role of VEGF-C staining in predicting regional metastasis in melanoma. Virchows Arch 453: 257-265, 2008.

35 Schietroma C, Cianfarani F, Lacal PM, Odorisio T, Orecchia A, Kanitakis J, D'Atri S, Failla CM and Zambruno G: Vascular endothelial growth factor-C expression correlates with lymph node localization of human melanoma metastases. Cancer 98 : 789-797, 2003.

36 Peppicelli S, Bianchini $\mathrm{F}$ and Calorini L: Inflammatory cytokines induce vascular endothelial growth factor-C expression in melanoma-associated macrophages and stimulate melanoma lymph node metastasis. Oncol Lett 8: 1133-1138, 2014.

37 Cianfarani F, Mastroeni S, Odorisio T, Passarelli F, Cattani C, Mannooranparampil TJ, Fortes C and Failla CM: Expression of vascular endothelial growth factor-C in primary cutaneous melanoma predicts sentinel lymph node positivity. J Cutan Pathol 39: 826-834, 2012.

38 Vihinen PP, Hilli J, Vuoristo MS, Syrjänen KJ, Kähäri VM and Pyrhönen SO: Serum VEGF-C is associated with metastatic site in patients with malignant melanoma. Acta Oncol 46: 678-684, 2007.

39 Raica M, Cimpean AM, Ceausu R, Ribatti D and Gaje P: Interplay between mast cells and lymphatic vessels in different molecular types of breast cancer. Anticancer Res 33: 957-963, 2013.

40 Raica M, Cimpean AM, Popovici RA, Balica AR, Vladau M and Gaje PN: Mast cells stimulate lymphangiogenesis in the gingiva of patients with periodontal disease. In Vivo 29: 29$34,2015$. 
41 Achen MG, Williams RA, Minekus MP, Thornton GE, Stenvers K, Rogers PA, Lederman F, Roufail $S$ and Stacker SA: Localization of vascular endothelial growth factor-D in malignant melanoma suggests a role in tumour angiogenesis. J Pathol 193: 147-154, 2001.

42 Špirić Z, Eri Ž and Erić M: Significance of Vascular Endothelial Growth Factor (VEGF)-C and VEGF-D in the progression of cutaneous melanoma. Int J Surg Pathol 23: 629-637, 2015.

43 Benest AV, Harper SJ, Herttuala SY, Alitalo K and Bates DO: VEGF-C induced angiogenesis preferentially occurs at a distance from lymphangiogenesis. Cardiovasc Res 78: 315-323, 2008.

44 Swoboda A, Schanab O, Tauber S, Bilban M, Berger W, Petzelbauer P and Mikula M: MET expression in melanoma correlates with a lymphangiogenic phenotype. Hum Mol Genet 21: 3387-3396, 2012.

45 Pastushenko I, Conejero C and Carapeto FJ: Lymphangiogenesis: implications for diagnosis, treatment, and prognosis in patients with melanoma. Actas Dermosifiliogr 106: 7-16, 2015.

46 Bracher A, Cardona AS, Tauber S, Fink AM, Steiner A, Pehamberger H, Niederleithner H, Petzelbauer P, Gröger M and Loewe R: Epidermal growth factor facilitates melanoma lymph node metastasis by influencing tumor lymphangiogenesis. J Invest Dermatol 133: 230-238, 2013.

47 Dadras SS: An unexpected role for EGF in lymphangiogenesismediated melanoma metastasis to sentinel lymph nodes. J Invest Dermatol 133: 14-16, 2013.

48 Mouawad R, Spano JP, Comperat E, Capron F and Khayat D: Tumoural expression and circulating level of VEGFR-3 (Flt-4) in metastatic melanoma patients: correlation with clinical parameters and outcome. Eur J Cancer 45: 1407-1414, 2009.

49 Mehnert JM, McCarthy MM, Jilaveanu L, Flaherty KT, Aziz S, Camp RL, Rimm DL and Kluger HM: Quantitative expression of VEGF, VEGF-R1, VEGF-R2, and VEGF-R3 in melanoma tissue microarrays. Hum Pathol 41: 375-384, 2010.

50 Rushing EC, Stine MJ, Hahn SJ, Shea S, Eller MS, Naif A, Khanna S, Westra WH, Jungbluth AA, Busam KJ, Mahalingam $\mathrm{M}$ and Alani RM: Neuropilin-2: a novel biomarker for malignant melanoma? Hum Pathol 43: 381-389, 2012.

51 Rossi M, Tuck J, Kim OJ, Panova I, Symanowski JT, Mahalingam M, Riker AI, Alani RM and Ryu B: Neuropilin-2 gene expression correlates with malignant progression in cutaneous melanoma. Br J Dermatol 171: 403-408, 2014.

52 Rinderknecht $\mathrm{M}$ and Detmar M: Tumor lymphangiogenesis and melanoma metastasis. J Cell Physiol 216: 347-354, 2008.

53 Pastushenko I, Van den Eynden GG, Vicente-Arregui S, PrietoTorres L, Alvarez-Alegret R, Querol I, Dirix LY, Carapeto FJ, Vermeulen PB and Van Laere SJ: Increased Angiogenesis and Lymphangiogenesis in Metastatic Sentinel Lymph Nodes Is Associated With Nonsentinel Lymph Node Involvement and Distant Metastasis in Patients With Melanoma. Am J Dermatopathol 38: 338-346, 2016.

54 Harrell MI, Iritani BM and Ruddell A: Tumor-induced sentinel lymph node lymphangiogenesis and increased lymph flow precede melanoma metastasis. Am J Pathol 170: 774-786, 2007.

55 Hirakawa S: From tumor lymphangiogenesis to lymphvascular niche. Cancer Sci 100: 983-989, 2009.
56 Liersch R, Hirakawa S, Berdel WE, Mesters RM and Detmar M: Induced lymphatic sinus hyperplasia in sentinel lymph nodes by VEGF-C as the earliest premetastatic indicator. Int J Oncol 41: 2073-2078, 2012.

57 Hoshida T, Isaka N, Hagendoorn J, di Tomaso E, Chen YL, Pytowski B, Fukumura D, Padera TP and Jain RK: Imaging steps of lymphatic metastasis reveals that vascular endothelial growth factor-C increases metastasis by increasing delivery of cancer cells to lymph nodes: therapeutic implications. Cancer Res 66: 8065-8075, 2006.

58 Lin J, Lalani AS, Harding TC, Gonzalez M, Wu WW, Luan B, Tu GH, Koprivnikar K, VanRoey MJ, He Y, Alitalo K and Jooss $\mathrm{K}$ : Inhibition of lymphogenous metastasis using adenoassociated virus-mediated gene transfer of a soluble VEGFR-3 decoy receptor. Cancer Res 65: 6901-6909, 2005.

59 Kinet V, Castermans K, Herkenne S, Maillard C, Blacher S, Lion M, Noël A, Martial JA and Struman I: The angiostatic protein $16 \mathrm{~K}$ human prolactin significantly prevents tumor-induced lymphangiogenesis by affecting lymphatic endothelial cells. Endocrinology 152: 4062-4071, 2011.

60 Duong T, Proulx ST, Luciani P, Leroux JC, Detmar M, Koopman $\mathrm{P}$ and Francois M: Genetic ablation of SOX18 function suppresses tumor lymphangiogenesis and metastasis of melanoma in mice. Cancer Res 72: 3105-3114, 2012.

61 Liersch R, Shin JW, Bayer M, Schwöppe C, Schliemann C, Berdel WE, Mesters R and Detmar M: Analysis of a novel highly metastatic melanoma cell line identifies osteopontin as a new lymphangiogenic factor. Int J Oncol 41: 1455-1463, 2012.

62 Niederleithner H, Heinz M, Tauber S, Bilban M, Pehamberger H, Sonderegger S, Knöfler M, Bracher A, Berger W, Loewe R and Petzelbauer P: Wnt1 is anti-lymphangiogenic in a melanoma mouse model. J Invest Dermatol 132: 2235-2244, 2012.

63 Roh MR, Zheng Z, Kim HS, Jeung HC, Rha SY and Chung KY: Difference of interferon- $\alpha$ and interferon- $\beta$ on melanoma growth and lymph node metastasis in mice. Melanoma Res 23: 114-124, 2013.

64 Heinz M, Niederleithner HL, Puujalka E, Soler-Cardona A, Grusch M, Pehamberger H, Loewe R and Petzelbauer P: Activin $\mathrm{A}$ is anti-lymphangiogenic in a melanoma mouse model. J Invest Dermatol 135: 212-221, 2015.

65 Kato Y1, Kaneko MK, Kuno A, Uchiyama N, Amano K, Chiba Y, Hasegawa Y, Hirabayashi J, Narimatsu H, Mishima K and Osawa M: Inhibition of tumor cell-induced platelet aggregation using a novel anti-podoplanin antibody reacting with its plateletaggregation-stimulating domain. Biochem Biophys Res Commun 349: 1301-1307, 2006.
Received July 15, 2016

Revised July 22, 2016

Accepted July 25, 2016 